\title{
IncRNA Expression Reveals the Potential Regulatory Roles in Hepatocyte Proliferation during Rat Liver Regeneration
}

\author{
Haijing Bai $\mathbb{D}^{1,2}$ Wei Jin $\mathbb{D}^{1,2}$, Jianlin Guo $\mathbb{D}^{1,2}$ Yi Ding $\mathbb{D}^{1,2}$ Cuifang Chang $\mathbb{D}^{1,2}$ \\ Xueqiang Guo $\mathbb{D},{ }^{1,2}$ Yaping Song $\mathbb{D}^{1,2}$ Jingbo Zhang $\mathbb{D}^{3}$, and Cunshuan $X u \mathbb{D}^{1,2}$ \\ ${ }^{1}$ College of Life Science, Henan Normal University, Xinxiang 453007, Henan Province, China \\ ${ }^{2}$ State Key Laboratory Cultivation Base for Cell Differentiation Regulation and Henan Engineering Laboratory \\ for Bioengineering and Drug Development, Xinxiang 453007, Henan Province, China \\ ${ }^{3}$ Institute of Basic Medical Sciences, Chinese Academy of Medical Sciences, Beijing 100005, China \\ Correspondence should be addressed to Jingbo Zhang; jingbozh@hotmail.com and Cunshuan Xu; cellkeylab@126.com
}

Received 10 June 2019; Revised 29 August 2019; Accepted 6 September 2019; Published 11 November 2019

Academic Editor: Monica Fedele

Copyright (c) 2019 Haijing Bai et al. This is an open access article distributed under the Creative Commons Attribution License, which permits unrestricted use, distribution, and reproduction in any medium, provided the original work is properly cited.

\begin{abstract}
Liver regeneration is a tissue growth process after loss or injury of liver tissue, which is a compensatory hyperplasia rather than true regeneration, mainly depending on hepatocyte proliferation. Currently, a large number of studies on hepatocyte proliferation have been conducted. However, studies on the regulation of long noncoding RNA (lncRNA) on hepatocyte proliferation are still limited. To identify specially expressed lncRNA during rat liver regeneration, high-throughput sequencing technology was performed, and a total of $2446 \mathrm{lncRNAs}$ and $4091 \mathrm{mRNAs}$ were identified as significantly differentially expressed. Gene ontology (GO) enrichment analysis was performed to analyze the role of differentially expressed mRNAs, and 695 mRNAs were identified to be related to cell proliferation. Then, an lncRNA-mRNA coexpression network based on the differentially expressed lncRNAs and proliferation-related genes was constructed to analyze the potential function of lncRNAs on hepatocyte proliferation, and ten lncRNAs, NONRATT003557.2, NONRATT005357.2, NONRATT003292.2, NONRATT001466.2, NONRATT003289.2, NONRATT001047.2, NONRATT005180.2, NONRATT004419.2, NONRATT005336.2, and NONRATT005335.2, were selected as key regulatory factors, which may play crucial roles in hepatocyte proliferation during rat liver regeneration. Finally, a protein-protein interaction (PPI) network was established to illuminate the interaction between proliferation-related genes, and ten hub genes (Aurkb, Cdk1, Cdc20, Bub1b, Mad211, Kif11, Prc1, Ccna2, Top2a, and Ccnb1) were screened with the MCC method in the PPI network, which may be important biomarkers involved in the hepatocyte proliferation during rat liver regeneration. These results may provide clues for a more comprehensive understanding of the molecular mechanism of hepatocyte proliferation during rat liver regeneration.
\end{abstract}

\section{Introduction}

The vast majority of the eukaryotic genomes are transcribed into noncoding RNAs, which can be divided into small noncoding RNAs $(<200 \mathrm{bp})$ and long noncoding RNAs (lncRNAs; $\geq 200 \mathrm{nt}$ ) based on transcript size [1]. lncRNAs can be divided into five categories: sense, antisense, bidirectional, intronic, and intergenic [2]. Initially, lncRNAs were considered to be "dark matter," as byproduct of transcription of RNA polymerase II, with no biological function. In the past 20 years, genome-wide identification of lncRNAs has become possible with the development of high-throughput technology of RNA-seq, many of which are involved in various biological functions [3]. Increasing lncRNAs have been found to play a critical role in biological processes, like development [4], gene transcriptional regulation [5], chromatin regulation [6], epithelialto-mesenchymal transition (EMT) [7], and cell proliferation [8].

In rodents and humans, the liver can grow rapidly after partial hepatectomy $(\mathrm{PH})$ or acute chemical injury. This growth process is known as LR, which is a compensatory hyperplasia rather than true regeneration [9]. During LR, quiescent hepatocytes undergo one or two rounds of 
replication and then return to a nonproliferative state [10]. This process is very complex and regulated by a variety of growth factors, cytokines and noncoding RNAs [11, 12]. Therefore, the study of the molecular mechanism of hepatocyte proliferation is crucially important to understand the process of LR and provide clues for the treatment of liver diseases. Several recent studies have shown that $\operatorname{lncRNAs}$ play a critical role in hepatocyte proliferation [12-14]. However, the study of hepatocyte proliferation during LR is still largely unknown.

In the present study, high-throughput sequencing technology was used to identify DE lncRNAs and mRNAs during rat LR. Then, functional enrichment analysis of DE mRNAs was performed to screen proliferation-related genes. Finally, the lncRNA-mRNA coexpression network and PPI network were constructed based on DE lncRNAs and proliferation-related genes to elucidate the molecular mechanism of hepatocyte proliferation during LR. These results lay a foundation for understanding the regulatory function of IncRNAs on hepatocyte proliferation and provide an important clue for the study of the LR process.

\section{Materials and Methods}

2.1. Preparation of $2 / 3$ Hepatectomy Model. The healthy adult male Sprague Dawley (SD) rats weighing 210 250 g were provided by the Laboratory Animal Center of Zhengzhou University (Zhengzhou, China). These rats were raised in a controlled temperature room of $19 \sim 23^{\circ} \mathrm{C}$ with a relative humidity of $50 \sim 70 \%$ and an illumination time of $12 \mathrm{~h} / \mathrm{d}(8: 00$ to $20: 00)$ and permitted to freely have water and food. A total of 60 rats were taken for the experiment with six rats per group: nine $\mathrm{PH}$ groups and one normal group (CG). The rats in $\mathrm{PH}$ groups were conducted $2 / 3 \mathrm{PH}$ according to the method of $\mathrm{Xu}$ et al. The rats were anesthetized and condemned to death at $0,2,6,12,24,30$, $36,72,120$, and $168 \mathrm{~h}$ after operation. The right liver lobes of six rats were mixed at each time point and stored at $-80^{\circ} \mathrm{C}$. All operations conformed to the Animal Protection Law of China and Animal Ethics.

2.2. RNA Sequencing. RNA sequencing was performed by the Shanghai OE Biotech (Shanghai, China). In brief, the mirVana miRNA Isolation Kit (Ambion) was used to extract the total RNA from liver tissues. The TruSeq Stranded Total RNA with Ribo-Zero Gold (Illumina) was used to construct cDNA libraries. The purified cDNA libraries were sequenced on Illumina HiSeq 2500 following the manufacturer's instruction. After filtrating the adaptor and low-quality reads, clean reads were obtained for subsequent analysis. The reads were matched to the rat reference genome using the hisat2 (v2.2.1.0) software. The StringTie2 (v1.3.3b) software was used to splice the aligned reads. IncRNA identification included two categories: one is known lncRNA, which completely matches with the known lncRNAs, and the other is candidate lncRNA lacking protein-coding ability, whose length is greater than $200 \mathrm{bp}$ and exon is greater than or equal to 2. The software CPC (v0.9-r2), CNCI (v1.0), Pfam (v30), and PLEK (v1.2) were used to predict the proteincoding ability of transcripts. The expression of transcription was calculated by the fragments per kilobase of exon per million reads mapped (FPKM) method using the bowtie2 (v2.2.9) and eXpress (v1.5.1) software.

2.3. Identification of Differentially Expressed IncRNAs. The counts of lncRNAs in each sample were standardized by the baseMean value using the DESeq (1.18.0) software. Differentially expressed (DE) lncRNAs were identified with fold change $\geq 2$ or $\leq 0.5$ and $p<0.05$ as the threshold. All DE lncRNAs in nine $\mathrm{PH}$ groups underwent hierarchical clustering analysis using the cluster3.0 and treeview software.

2.4. GO Enrichment Analysis. Gene ontology (GO) enrichment analysis of the DE mRNAs was conducted using David Bioinformatics Resources 6.8 (https://david.ncifcrf.gov/). The enrichment analysis consisted of three parts: biological process (BP), molecular function (MF), and cellular component (CC). $p<0.05$ is considered statistically significant, which was calculated by the EASE score.

2.5. IncRNA-mRNA Coexpression Analysis. To explore the relationship between DE lncRNAs and proliferation-related genes, a coexpression analysis was performed. Pearson's correlation coefficients (PCCs) were calculated between the DE lncRNAs and the proliferation-related genes, and only lncRNA-mRNA pairs with PCC $\geq 0.8$ and $p \leq 0.05$ were selected and considered as coexpression. Then, these IncRNA-mRNA pairs were used to construct a coexpression network, which was visualized by the Cytoscape v3.6.1 software. The node degree was determined by the number of directly connected neighbors to the topological property of the network.

2.6. Construction of PPI Network and Screening of the Key Gene. To illustrate interactions between proliferation-related DE mRNAs, the string database (https://string-db.org/) was used to construct a protein-protein interaction (PPI) network. Only the interacting pairs with combined score $\geq 0.4$ were selected and considered to be significant. The PPI network was visualized by using the Cytoscape v3.6.1 software. Then, a Cytoscape plugin cytoHubba was used to identify the key genes adopting the MCC method.

2.7. RT-PCR Validation. RNA-seq results were validated by RT-PCR, and the primers are listed in Table 1. cDNA was synthesized using the cDNA Reverse Transcription Kit (Takara, Tokyo, Japan). The qRT-PCR was performed using Q-SYBR Green Supermix (Bio-Rad). Primers were also designed to amplify $\beta$-actin as an endogenous control. The expression of each lncRNA was represented as fold change using $2^{-\Delta \Delta \mathrm{Ct}}$ methods. 
TABLe 1: Primer sequence.

\begin{tabular}{lccc}
\hline Gene name & Sense primer & Antisense primer & Product length \\
\hline NONRATT003289.2 & AATGCCAGGCCATGCTAAGGAC & GCTCTGCCAGGTGACTGCTTC & 180 \\
NONRATT001466.2 & TCTGCTGTTGACATTGGCGAAGG & CTAGCATGTGAGAGGTGACGTGAC & 189 \\
NONRATT004419.2 & AGCCTCCTGAGTCCTGGAATTCTG & GTGAGTCGTGAGTGAGCTGAAGTG & 133 \\
NONRATT005336.2 & AAGCTCAACACTGCCTGAGTCTTC & GCATGAGCCTTGGAGGACATCTG & 112 \\
\hline
\end{tabular}

Table 2: Overview of RNA-seq.

\begin{tabular}{lccccccc}
\hline Sample & raw_reads & raw_bases & clean_reads & clean_bases & valid_bases (\%) & GC (\%) & Mapped reads \\
\hline $0 \mathrm{~h}$ & $119.65 \mathrm{M}$ & $17.95 \mathrm{G}$ & $115.99 \mathrm{M}$ & $17.05 \mathrm{G}$ & 94.99 & 50.92 & $111716504(96.32 \%)$ \\
$2 \mathrm{~h}$ & $120.14 \mathrm{M}$ & $18.02 \mathrm{G}$ & $116.69 \mathrm{M}$ & $17.17 \mathrm{G}$ & 95.25 & 50.57 & $113657852(97.40 \%)$ \\
$6 \mathrm{~h}$ & $119.57 \mathrm{M}$ & $17.94 \mathrm{G}$ & $116.13 \mathrm{M}$ & $17.09 \mathrm{G}$ & 95.29 & 50.26 & $113238482(97.51 \%)$ \\
$12 \mathrm{~h}$ & $119.91 \mathrm{M}$ & $17.99 \mathrm{G}$ & $117.68 \mathrm{M}$ & $16.68 \mathrm{G}$ & 92.75 & 49.88 & $114912884(97.65 \%)$ \\
$24 \mathrm{~h}$ & $119.27 \mathrm{M}$ & $17.89 \mathrm{G}$ & $117.28 \mathrm{M}$ & $16.23 \mathrm{G}$ & 90.73 & 48.77 & $114629815(97.74 \%)$ \\
$30 \mathrm{~h}$ & $88.95 \mathrm{M}$ & $13.34 \mathrm{G}$ & $85.96 \mathrm{M}$ & $12.64 \mathrm{G}$ & 94.74 & 50.29 & $83564746(97.21 \%)$ \\
$36 \mathrm{~h}$ & $120.15 \mathrm{M}$ & $18.02 \mathrm{G}$ & $116.44 \mathrm{M}$ & $17.16 \mathrm{G}$ & 95.22 & 50.62 & $113908105(97.83 \%)$ \\
$72 \mathrm{~h}$ & $91.48 \mathrm{M}$ & $13.72 \mathrm{G}$ & $88.07 \mathrm{M}$ & $12.9 \mathrm{G}$ & 93.98 & 51.08 & $86032773(97.68 \%)$ \\
$120 \mathrm{~h}$ & $120.82 \mathrm{M}$ & $18.12 \mathrm{G}$ & $116.34 \mathrm{M}$ & $17.12 \mathrm{G}$ & 94.47 & 50.97 & $112635056(96.82 \%)$ \\
$168 \mathrm{~h}$ & $96.06 \mathrm{M}$ & $14.41 \mathrm{G}$ & $93.51 \mathrm{M}$ & $13.73 \mathrm{G}$ & 95.28 & 50.71 & $89681397(95.91 \%)$ \\
\hline
\end{tabular}

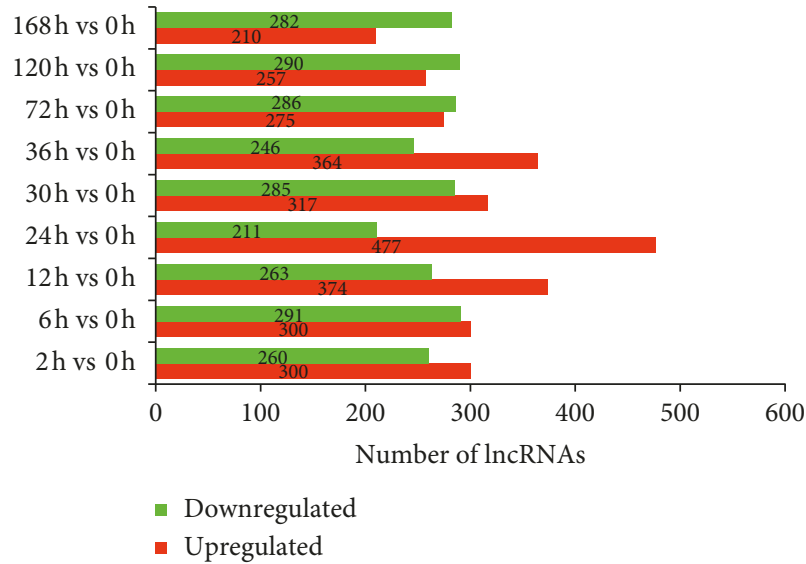

(a)

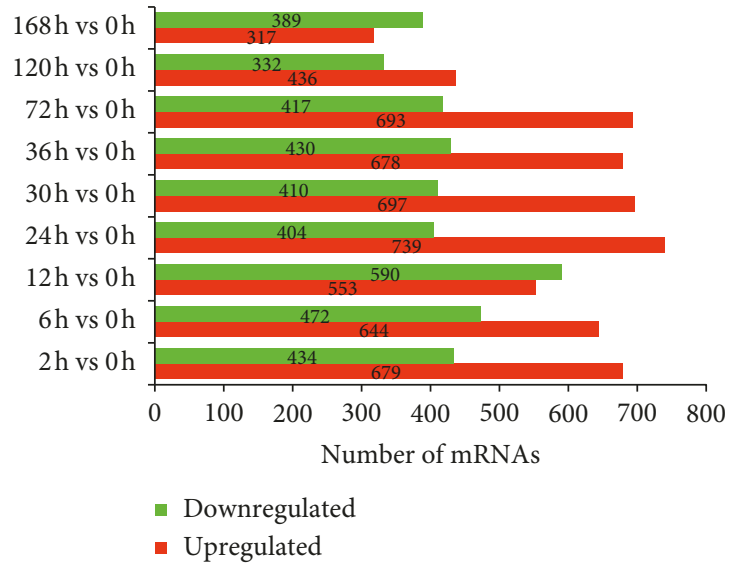

(b)

Figure 1: Number of DE lncRNAs and DE mRNAs at different time points during rat LR. (a) Number of upregulated and downregulated lncRNAs at nine time points. (b) Number of upregulated and downregulated mRNAs at nine time points.

\section{Results}

3.1. Sequencing and Identification of $\operatorname{lnc} R N A$ s during Rat $L R$. To identify the expression of lncRNAs during rat LR, 10 cDNA libraries were constructed from the regeneration rat liver at different time points after surgery. The Illumina HiSeq X Ten platform was used to sequence these cDNA libraries, and a total of $1116 \mathrm{M}$ raw reads were produced. After filtering adaptor sequences and low-quality reads, 1084.09M clean reads were obtained. The percentage of clean reads varied from 90.73 to $95.29 \%$, and the percentage of GC content varied from 48.77 to $51.08 \%$ in each library. Approximately $95.91-97.83 \%$ of clean reads were selected for further research after mapping the clean reads to the rat reference genome (Table 2).

3.2. Identification of DE $\operatorname{lncRNAs}$ and $D E m R N A s$. The expression abundance of the lncRNAs was evaluated by FPKM (fragments per $\mathrm{kB}$ per million reads) using DESeq. A total of 2446 lncRNAs were determined to be differentially expressed during rat LR, with 1120 upregulated, 731 downregulated, and 595 up/downregulated lncRNAs (Figure 1(a) and Table S1). To explore the similarity of gene expression, hierarchical clustering was adopted to analyze the expression of DE lncRNAs (Figure 2(a)). To further explore the interactions of DE lncRNAs at different stages: initial stage $(2-6 \mathrm{~h})$, proliferation stage (12-72 h), and termination stage (120-168h), a Venn diagram was constructed using these DE lncRNAs (Figure 2(c)). Among them, 272 DE lncRNAs were common to all three stages.

Through high-throughput RNA-seq, the expression profile of 28635 mRNAs was measured. Among them, 4091 mRNAs were found to be differentially expressed, of which 2,256 were upregulated, 1,686 were downregulated, and 149 were up/downregulated (Figure 1(b) and Table S2). Hierarchical clustering was employed to analyze the expression 


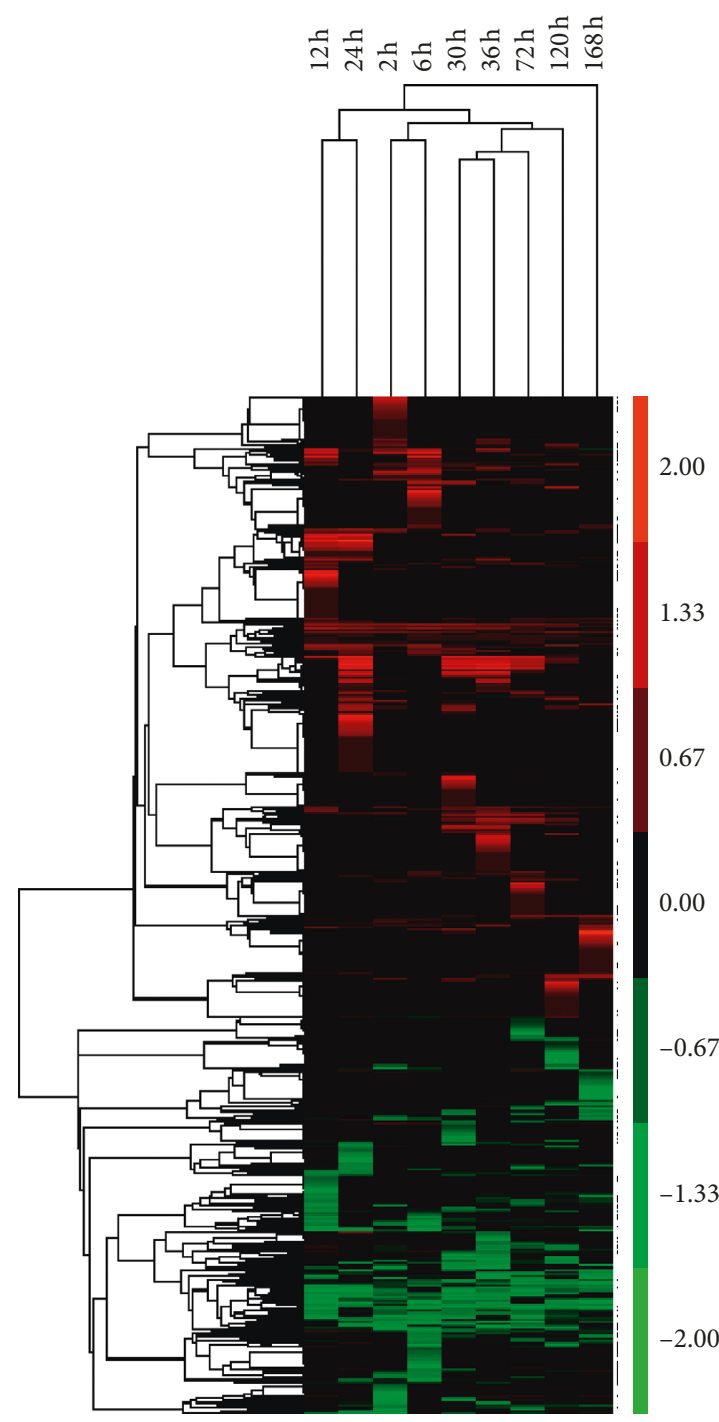

(a)

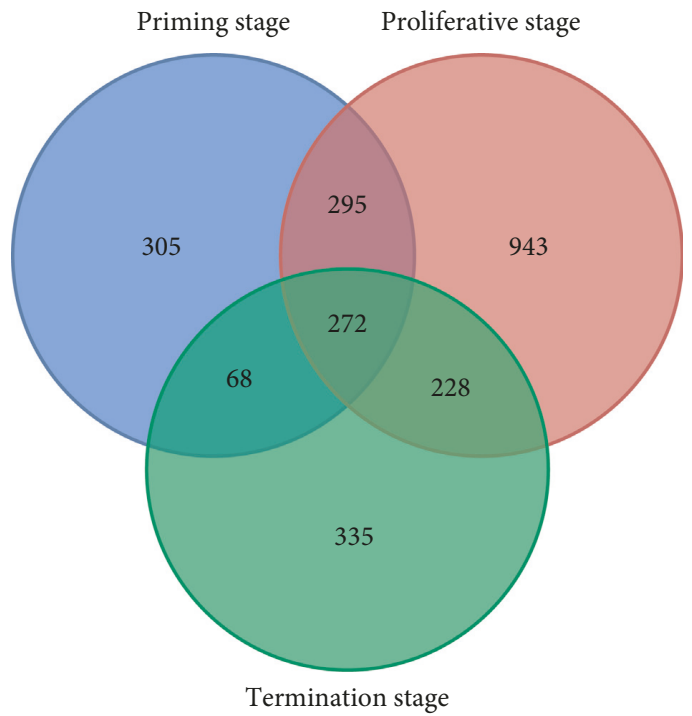

(c)

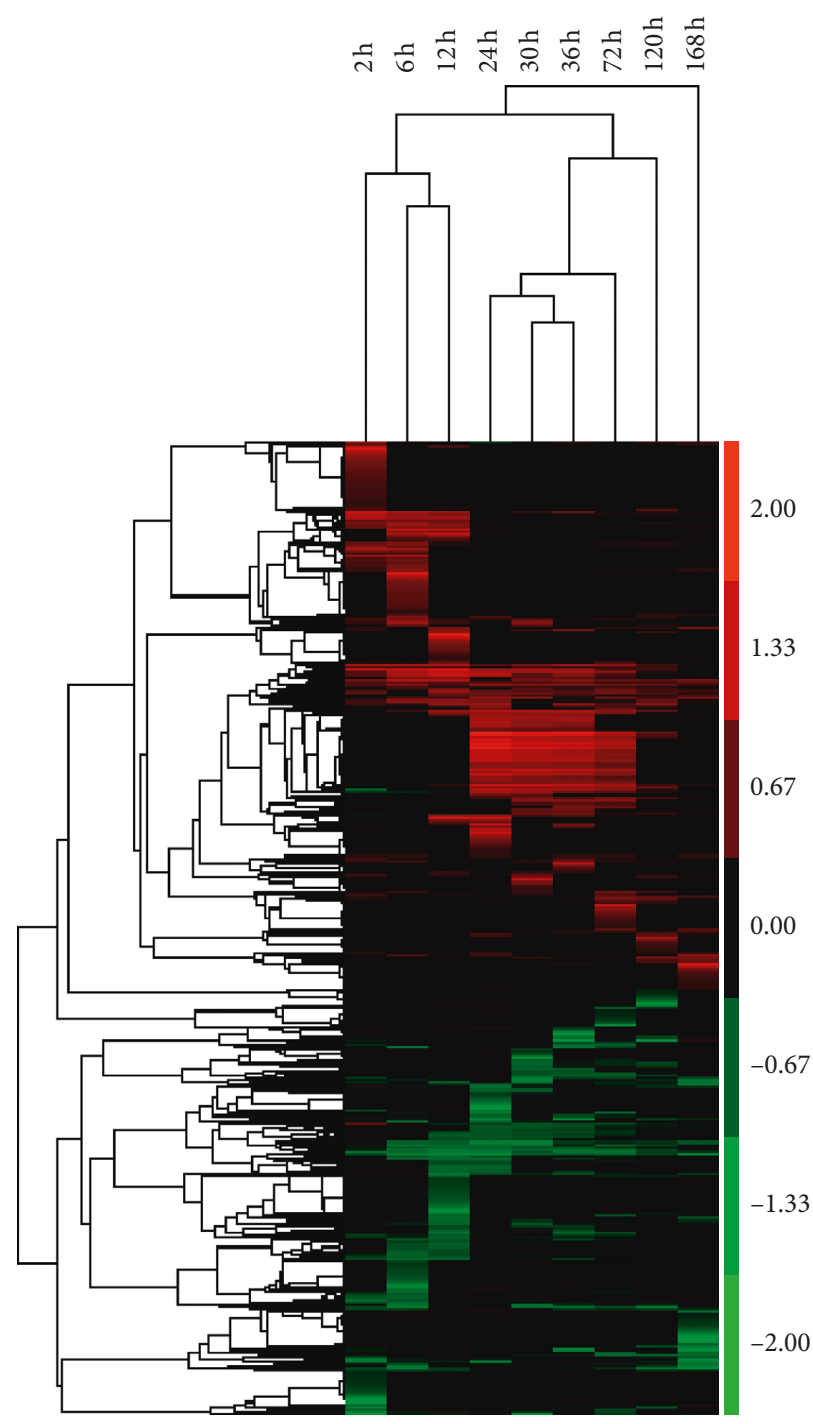

(b)

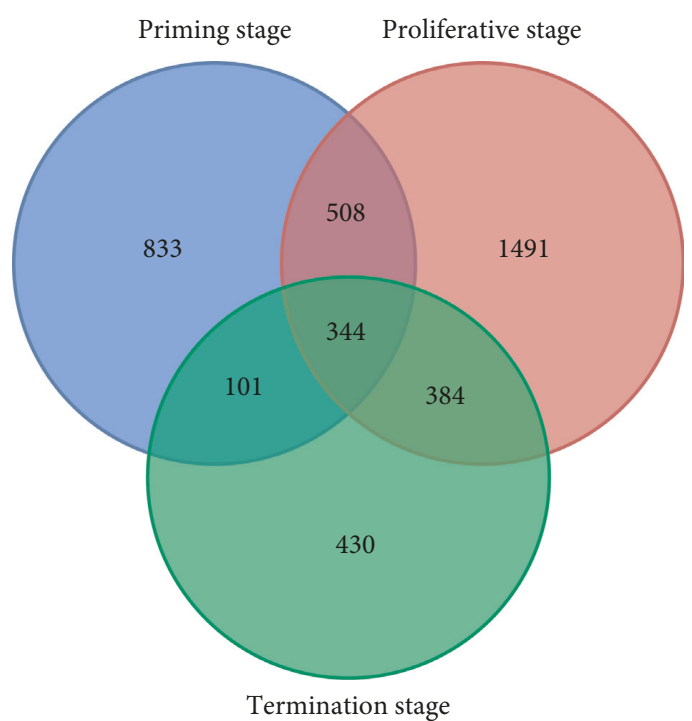

(d)

FIgURE 2: Analyses of DE lncRNAs in the RNA-seq libraries. (a) Hierarchical clustering analysis of DE lncRNAs at nine time points of rat LR. (b) Hierarchical clustering analysis of DE mRNAs at nine time points of rat LR. (c) Venn diagram showing the DE lncRNAs at three stages of rat LR. (d) Venn diagram showing the DE mRNAs at three stages of rat LR. 


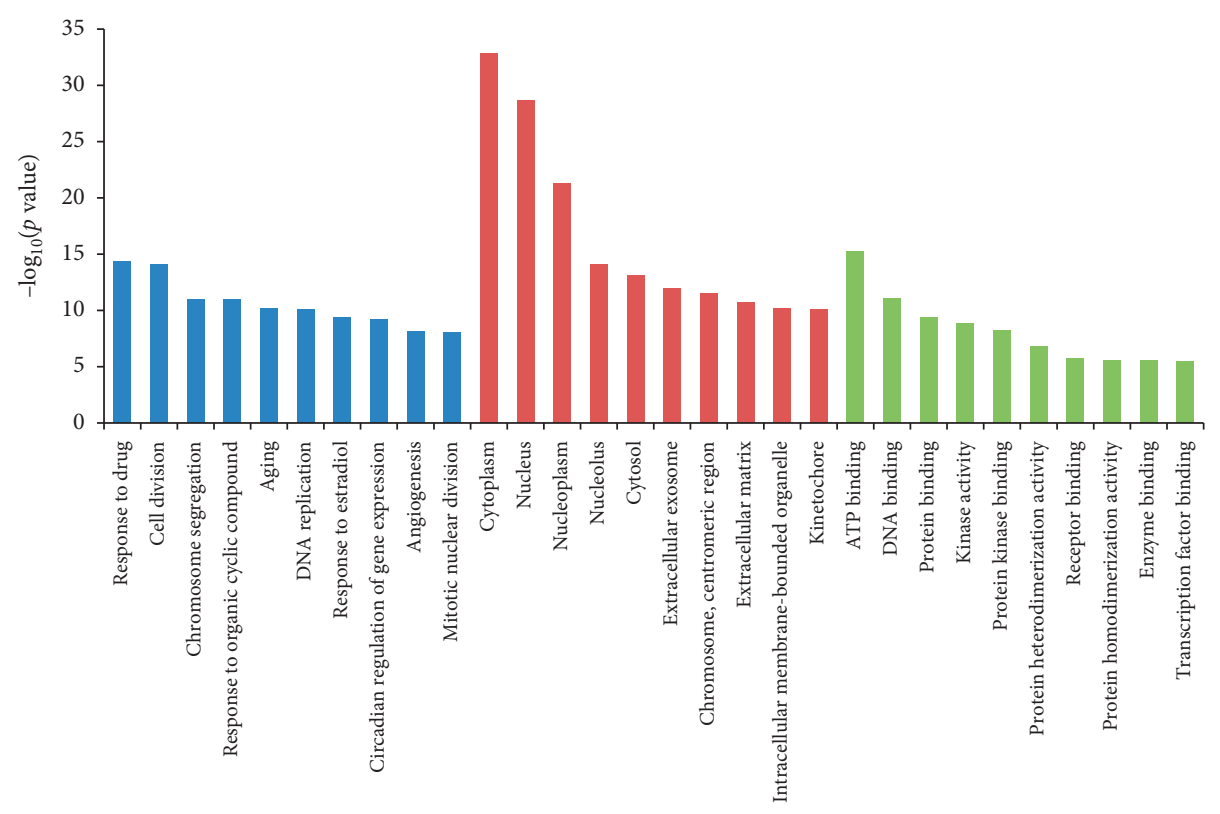

- Biological process

- Cellular component

- Molecular function

(a)

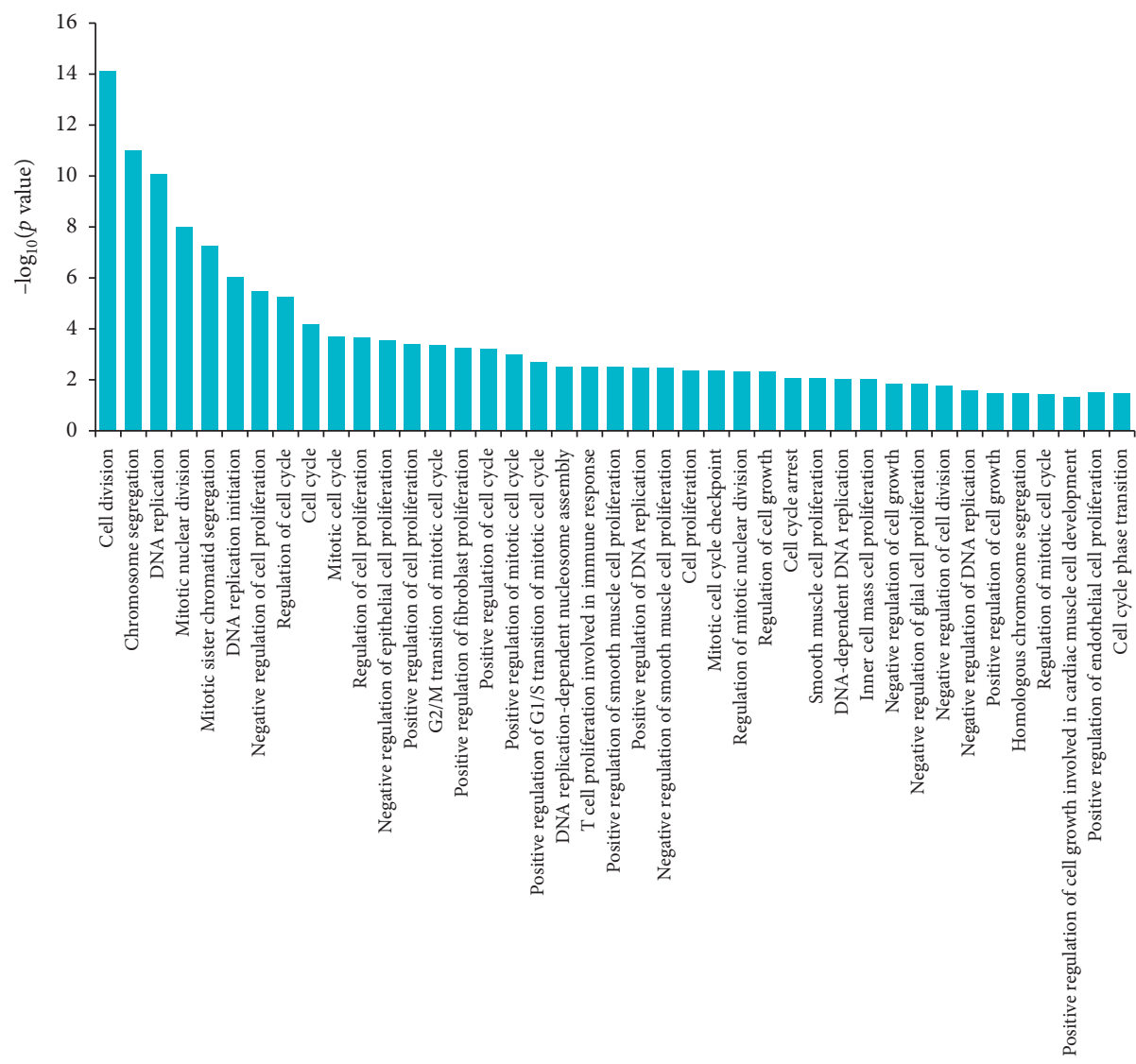

(b)

Figure 3: GO enrichment analysis of the DE mRNAs. (a) The top 30 significant GO terms in GO enrichment analysis at $p$ value $<0.05$. Green represents biological processes, red represents cellular components, and blue represents molecular functions. (b) GO terms associated with cell proliferation at the biological process level. 


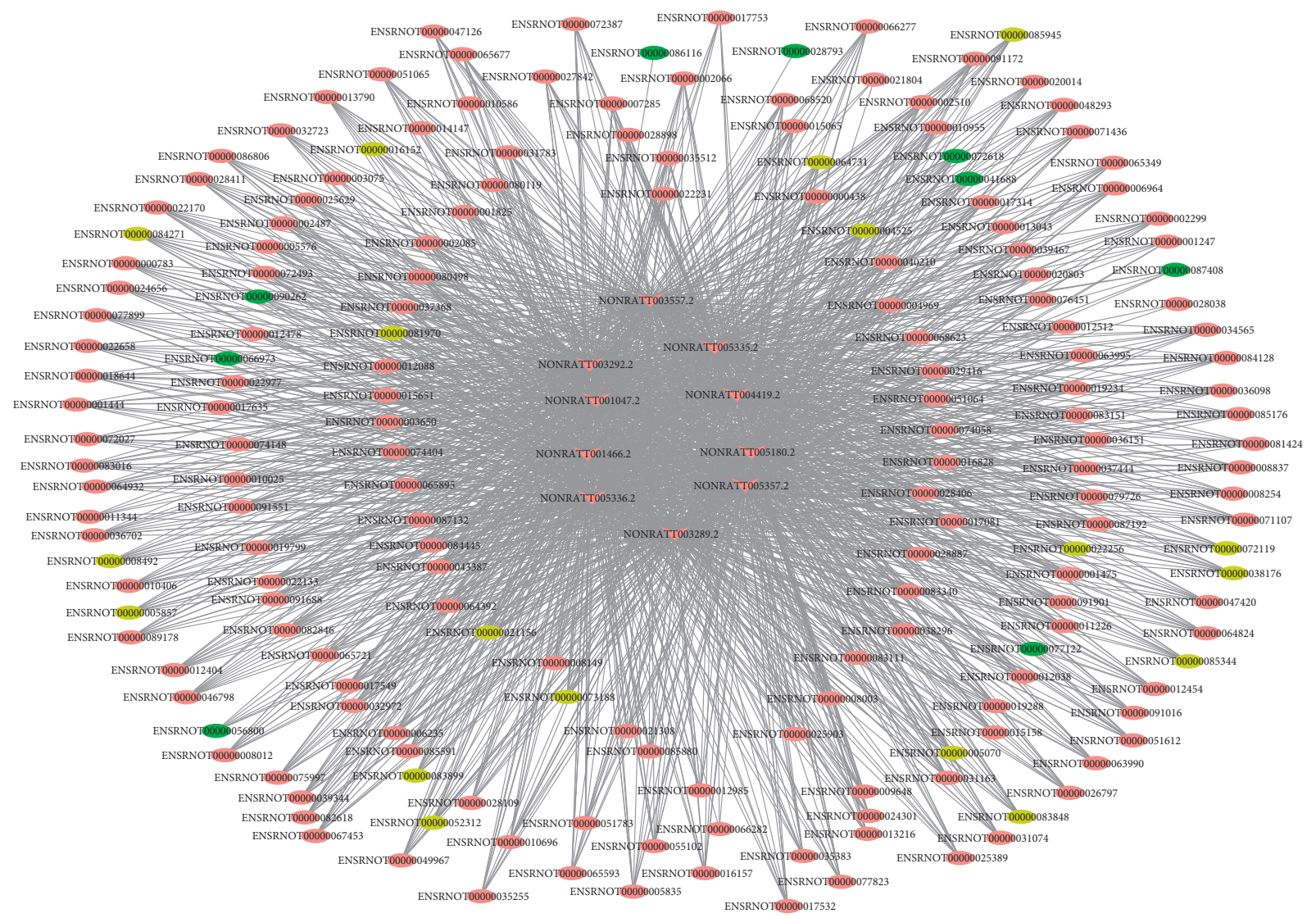

FIGURE 4: The top 10 lncRNAs and their interaction mRNAs. V and ellipse represent lncRNAs and mRNAs, respectively. Upregulated genes are labeled in red, downregulated genes are labeled in green, and up/downregulated genes are labeled in yellow.

TABLE 3: Expression levels of the top $10 \operatorname{lncRNAs}$ during rat LR.

\begin{tabular}{|c|c|c|c|c|c|c|c|c|c|c|}
\hline lncRNAs & $0 \mathrm{~h}$ & $2 \mathrm{~h}$ & $6 \mathrm{~h}$ & $12 \mathrm{~h}$ & $24 \mathrm{~h}$ & $30 \mathrm{~h}$ & $36 \mathrm{~h}$ & $72 \mathrm{~h}$ & $120 \mathrm{~h}$ & $168 \mathrm{~h}$ \\
\hline NONRATT003557.2 & 1 & 1 & 1 & 1 & 14.67 & 15.41 & 10.44 & 1 & 1 & 1 \\
\hline NONRATT005357.2 & 1 & 1 & 1 & 9.79 & 119.12 & 59.69 & 71.42 & 32.46 & 1 & 1 \\
\hline NONRATT003292.2 & 1 & 1 & 1 & 1 & 43.68 & 40.42 & 28.50 & 18.78 & 1 & 1 \\
\hline NONRATT001466.2 & 1 & 1 & 1 & 1 & 20.37 & 23.28 & 24.66 & 13.95 & 1 & 1 \\
\hline NONRATT003289.2 & 1 & 1 & 1 & 1 & 69.44 & 53.97 & 38.69 & 23.26 & 6.55 & 1 \\
\hline NONRATT001047.2 & 1 & 1 & 1 & 1 & 1 & 1 & 4.86 & 1 & 1 & 1 \\
\hline NONRATT005180.2 & 1 & 1 & 1 & 1 & 9.81 & 9.02 & 10.33 & 1 & 1 & 1 \\
\hline NONRATT004419.2 & 1 & 1 & 1 & 1 & 1 & 6.87 & 6.87 & 7.5 & 1 & 1 \\
\hline NONRATT005336.2 & 1 & 1 & 1 & 1 & 1 & 6.83 & 1 & 1 & 1 & 1 \\
\hline NONRATT005335.2 & 1 & 1 & 1 & 1 & 6.89 & 6.44 & 5.27 & 1 & 1 & 1 \\
\hline
\end{tabular}

Text in bold denotes the expression level higher than the control.

similarity of DE mRNAs (Figure 2(b)). Venn analysis was conducted to explore the differences of DE mRNAs at different stages (Figure 2(d)).

3.3. GO and KEGG Enrichment Analysis. GO enrichment analysis was employed to determine the function of deregulated genes during rat LR, and 689 significant GO terms (451 under BP, 116 under CC, and 122 under MF) were enriched. The top $30 \mathrm{GO}$ terms of the three groups are listed in Figure 3(a) (Table S3). The most enriched BP terms were response to drug, cell division, and chromosome segregation. As for CC, the most enriched terms were cytoplasm, nucleus, and nucleoplasm. The most enriched MF terms were related to binding activity. Of these BP terms, 41 were associated with cell proliferation involving 695 mRNAs, corresponding to 585 genes (Figure $3(\mathrm{~b})$ and Table S3).

3.4. Coexpression Network Construction Based on DE IncRNAs and Proliferation-Related DE mRNAs. The coexpression analysis of the screened proliferation-related DE mRNAs and DE lncRNAs found 18343 significant coexpression pairs, of which 17547 pairs were positively correlated $(\mathrm{PCC} \geq 0.8)$ and 796 pairs were negatively correlated 


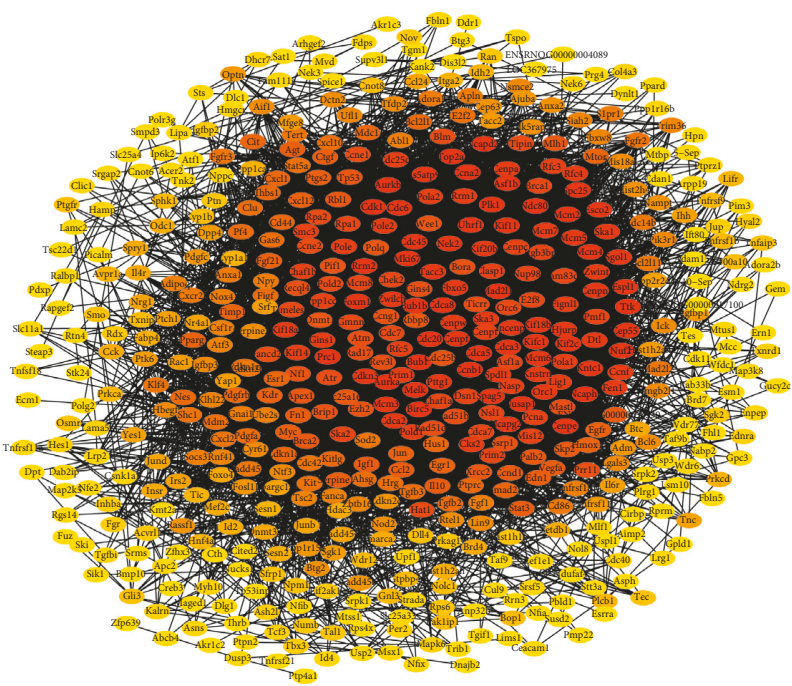

(a)

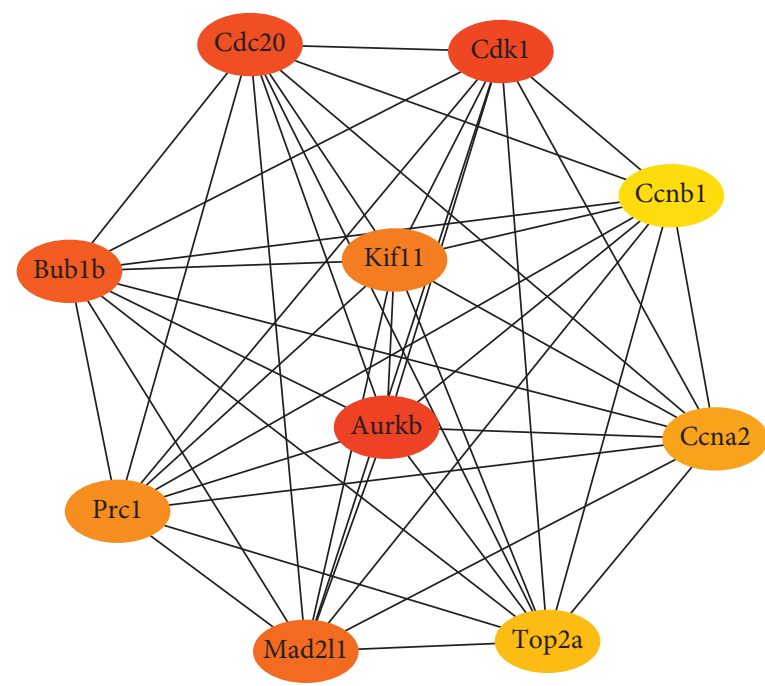

(b)

FIgURE 5: PPI network of proliferation-related genes. (a) PPI network of 551 genes. (b) Top 10 hub DE mRNAs of the PPI network. The node color changes gradually from yellow to red in the ascending order according to scores with the MCC method.

TABLE 4: Expression levels of 10 key genes during rat LR.

\begin{tabular}{|c|c|c|c|c|c|c|c|c|c|c|}
\hline Genes & $0 \mathrm{~h}$ & $2 \mathrm{~h}$ & $6 \mathrm{~h}$ & $12 \mathrm{~h}$ & $24 \mathrm{~h}$ & $30 \mathrm{~h}$ & $36 \mathrm{~h}$ & $72 \mathrm{~h}$ & $120 \mathrm{~h}$ & $168 \mathrm{~h}$ \\
\hline Aurkb & 1 & 0.02 & 1 & 1 & 41.88 & 26.57 & 23.96 & 14.00 & 3.72 & 1 \\
\hline Cdk1 & 1 & 1 & 1 & 1 & 109.01 & 66.43 & 80.87 & 42.31 & 3.63 & 1 \\
\hline $\mathrm{Cdc} 20$ & 1 & 1 & 1 & 1 & 36.85 & 27.36 & 25.84 & 13.01 & 2.83 & 1 \\
\hline Bublb & 1 & 1 & 1 & 1 & 38.34 & 26.78 & 22.37 & 12.92 & 5.08 & 1 \\
\hline Mad2l1 & 1 & 1 & 1 & 1 & 20.26 & 13.71 & 13.11 & 7.02 & 1 & 1 \\
\hline Kif11 & 1 & 1 & 1 & 1 & 20.48 & 13.24 & 11.22 & 7.31 & 2.12 & 1 \\
\hline Prcl & 1 & 1 & 1 & 1 & 46.88 & 29.75 & 27.15 & 16.27 & 3.59 & 1 \\
\hline Ccna2 & 1 & 0.05 & 1 & 1 & 49.97 & 31.85 & 30.16 & 15.45 & 3.92 & 1 \\
\hline Ccnb1 & 1 & 1 & 1 & 1 & 60.31 & 37.31 & 37.82 & 18.90 & 3.45 & 1 \\
\hline Top2a & 1 & 0.18 & 1 & 1 & 49.62 & 34.69 & 23.24 & 14.75 & 3.03 & 1 \\
\hline
\end{tabular}

Text in bold and text in italics denote the expression level higher and lower than the control, respectively.

(PCC $\leq-0.8)$ (Table S4). Subsequently, a coexpressed network was constructed using screened lncRNA-mRNA pairs, and it was found that some lncRNAs could interact with multiple mRNAs. According to nodes and connectivity, the top 10 lncRNAs were selected, which may exert important regulatory roles in hepatocyte proliferation, lncRNAs NONRATT003557.2 (degree $=178)$, NONRATT005357.2 $($ degree $=178)$, NONRATT003292.2 $($ degree $=177)$, NONRATT001466.2 $\quad($ degree $=176), \quad$ NONRATT003289.2 $($ degree $=176)$, NONRATT001047.2 $($ degree $=175)$, NONRATT005180.2 $\quad($ degree $=175), \quad$ NONRATT004419.2 $($ degree $=173), \quad$ NONRATT005336.2 $\quad($ degree $=173), \quad$ and NONRATT005335.2 $($ degree $=169) \quad($ Figure 4$)$. The expression levels of the top 10 lncRNAs during rat LR are shown in Table 3. Among them, NONRATT005357.2 had the highest difference at $24 \mathrm{~h}$ in regenerating the liver, and the fold change was 119 .

3.5. Construction of PPI Network Based on Proliferation-Related Genes. To better elucidate the interaction network of proliferation-related genes, a PPI network containing 551 nodes with scores greater than or equal to 0.4 was constructed using the string database (Figure 5(a)). Moreover, a cytoHubba plugin was used to select the top 10 key genes from the PPI network using the MCC method. The 10 key genes were Aurkb, Cdk1, Cdc20, Bub1b, Mad211, Kif11, Prc1, Ccna2, Top2a, and Ccnb1 (Figure 5(b)). The expression levels of the 10 key genes during rat LR are shown in Table 4 . The median of multiple mRNAs corresponding to one gene is taken as its expression level. Among them, Cdk1 had the highest difference at $24 \mathrm{~h}$ in regenerating the liver, and the fold change was 109.

3.6. Quantitative Real-Time PCR Validation. We validated the high-throughput RNA-seq results by performing qRTPCR analysis of differentially expressed lncRNAs; the expression patterns revealed similar conclusions (Figure 6). Our RNA-seq results showed lncRNAs NONRATT003289.2, NONRATT001466.2, NONRATT004419.2, and NONRATT005336.2 were upregulated during rat LR. 
NONRATT001466.2

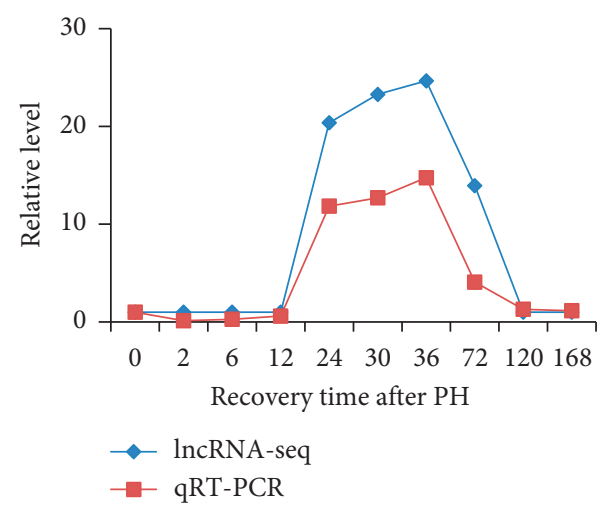

(a)

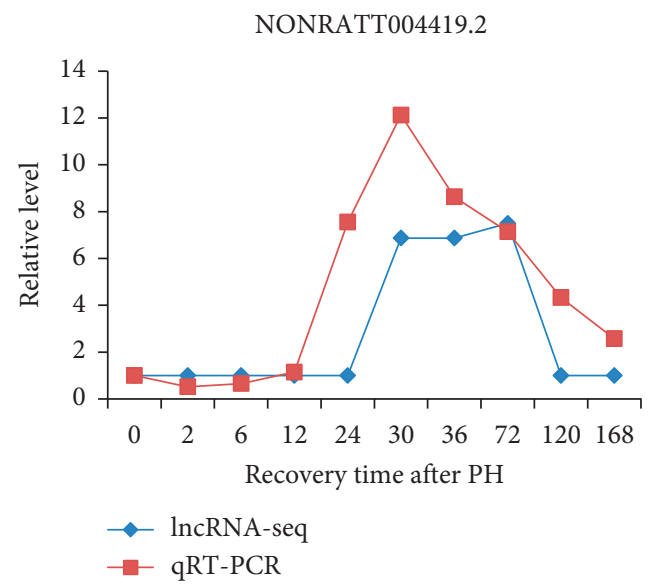

(c)

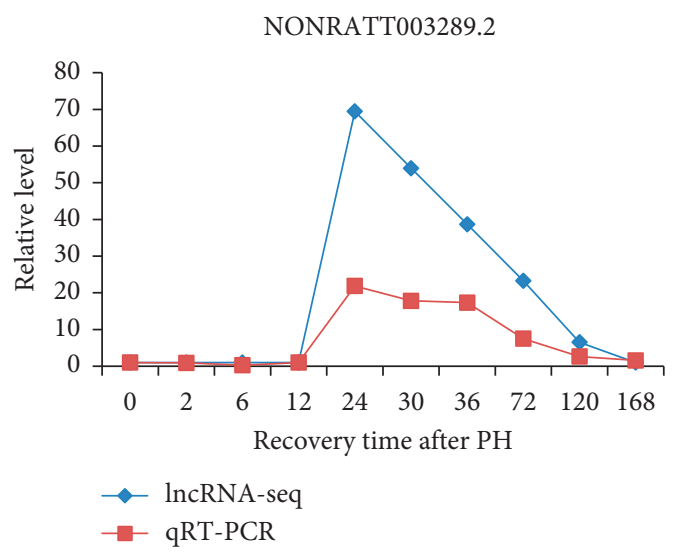

(b)

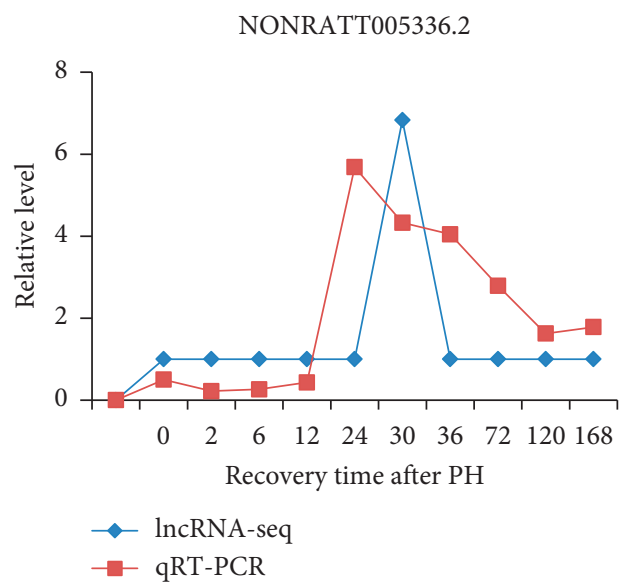

(d)

FIGURE 6: Validation of differentially expressed lncRNAs by qRT-PCR.

\section{Discussion}

In rodents and humans, the liver can grow rapidly after partial hepatectomy or acute chemical injury. This growth process is known as LR, which is a compensatory hyperplasia rather than true regeneration, mainly depending on the proliferation of hepatocytes [9]. To explore the regulatory roles of lncRNAs in hepatocyte proliferation during rat LR, high-throughput RNA-seq was performed to identify lncRNAs and mRNAs. In the present research, $2446 \mathrm{DE}$ lncRNAs and 4091 DE mRNAs were identified. To investigate the function of $\mathrm{DE}$ mRNAs during rat LR, GO enrichment analysis was performed. The result indicated that a large number of GO terms were associated with response to stress, cell proliferation, oxidation-reduction, regulation of transcription, metabolism, and apoptosis, which were considered to be important activities in LR [15-17]. Of these GO terms, 41 were associated with cell proliferation, involving 695 mRNAs.

Through coexpression network analysis, a total of 18343 coexpression pairs were obtained based on DE lncRNAs and proliferation-related DE mRNAs. Among these coexpression pairs, $95.7 \%$ were positively correlated and $4.3 \%$ were negatively correlated. These results indicate that lncRNAs may regulate the expression of genes mainly in a positive way. It was also found that some lncRNAs could be coexpressed with multiple mRNAs, suggesting that lncRNAs could regulate multiple mRNAs. According to nodes and connections, the top 10 lncRNAs (NONRATT003557.2, NONRATT005357.2, NONRATT003292.2, NONRATT001466.2, NONRATT003289.2, NONRATT001047.2, NONRATT005180.2, NONRATT004419.2, NONRATT005336.2, and NONRATT005335.2) were selected. These lncRNAs were connected to 158 common DE mRNAs, respectively, such as Slc25a16, Cdk1, Rfc3, Brca2, Mcm7, Cdca7, Hat1, Chaf1b, $\mathrm{Rfc4}$, and $\mathrm{Mcm} 4$. These findings indicated that these IncRNAs and their interaction genes may play an important role in hepatocyte proliferation during rat LR.

cytoHubba, a Cytoscape plugin, could provide 11 topological analysis methods to explore important nodes in biological networks including maximal clique centrality (MCC). MCC, a new method, has a better precision in identifying hub proteins. Through the MCC method, 10 hub mRNAs were selected including Aurkb, Cdk1, Cdc20, Bub1b, Mad211, Kif11, Prc1, Ccna2, Top2a, and Ccnb1. Aurkb (aurora kinase B) was involved in regulation of chromatin, glycolysis metabolism, regulation of telomerase activity, regulation of zygote development, and turnover of kinetochore microtubules [18-22]. It was also associated with AKT signaling pathway, MAPK signaling 
pathway, inflammatory pathway, DNA damage response pathway, Wnt signaling pathway, and PI3K/Akt/NF- $\kappa \mathrm{B}$ signaling pathway $[21,23-27]$. Cdk1 (cyclin-dependent kinase 1) was involved in cell cycle, mitosis, DNA end resection, homologous recombination, DNA damage checkpoint, anaphase spindle dynamics, cytokinesis, chromosome biorientation, apoptosis, cell proliferation and survival, and DNA synthesis [28-35]. It also played an important role in AMPK signaling pathway, AKT signaling pathway, Golgi checkpoint signaling, PDK1-PI3K/Akt signaling pathway, type I interferon signaling, and p53 signaling pathway [36-40]. Cdc20 (cell division cycle 20), an APC activator protein, could regulate mitosis, cell cycle, and presynaptic differentiation [41-44]. It was also involved in Wnt/ $\beta$-catenin signaling pathway and p38 MAPK signaling pathway. Bub1b (BUB1 mitotic checkpoint serine/threonine kinase B) and Mad2l1 (mitotic arrest deficient 2 like 1) were involved in the spindle checkpoint during mitosis [45-48]. Kif11 (kinesin family member 11), a microtubule motor, played a vital role in regulating the transport of $\beta$-actin mRNA and cell motility through physically interacting with ZBP1, which could govern the direction of migration by responding to directional cues in chemotaxis $[49,50]$. Prc1 (protein regulator of cytokinesis 1), a cell cycle protein, played an important role in ensuring proper cell division through directly acting on protein (FIP), and P53 could regulate the transcription of $\operatorname{Prc1}[51,52]$. Ccna2 (cyclin A2) was a key factor in cell cycle, and $\mathrm{Ccna} 2$ repression regulated by miR-22 could inhibit HCC cell proliferation and tumorigenesis $[53,54]$. It was also a prognostic biomarker for several cancers, such as ER+ breast cancer, pancreatic ductal adenocarcinoma, and colorectal cancer [55-57]. Top2a (DNA topoisomerase II alpha), a key enzyme in DNA replication, was recruited to ultrafine anaphase bridges (UFBs) by TopBP1 to ensure faithful separation of sister chromatids, and these proteins played an important role in maintaining genome stability [58]. Top2a was overexpressed in hepatocellular carcinoma and associated with early age onset, shorter patient survival, and chemoresistance [59]. Ccnb1 (cyclin B1) was a key factor in cell cycle and regulated by STAT3 via the E2F modulating G2-M phase checkpoint [60]. High expression of Ccnb1 was associated with poor prognosis in HCC patients, and knockdown of Ccnb1 could significantly inhibit cell proliferation, migration, and invasion in HCC [61]. Therefore, these genes may play a vital role in hepatocyte proliferation during LR.

\section{Conclusions}

In this study, the comprehensive expression abundance of lncRNAs and mRNAs was identified by RNA-seq analysis during rat LR. The lncRNA-mRNA coexpression network and PPI network based on lncRNAs and proliferation-related genes were constructed, and $10 \mathrm{key}$ lncRNAs and 10 key mRNAs were determined that may play crucial roles in hepatocyte proliferation. Our study provides a new idea to better understand the mechanism of LR.

\section{Data Availability}

The data used to support the findings of this study are included within the supplementary information file(s).

\section{Conflicts of Interest}

The authors declare that there are no conflicts of interest.

\section{Acknowledgments}

This study was financially supported by the National Natural Science Foundation of China (no. 31572270) and the National Natural Science Foundation of China Youth Science Fund Project (no. 31601038).

\section{Supplementary Materials}

Table S1: results of analyzing differentially expressed lncRNAs at 2, 6, 12, 24, 30, 36, 72,120, and $168 \mathrm{~h}$ after $\mathrm{PH}$ was compared with CG. Table S2: results of analyzing differentially expressed mRNAs at 2, 6, 12, 24, 30, 36, 72,120, and $168 \mathrm{~h}$ after $\mathrm{PH}$ was compared with CG. Table S3: results of GO enrichment analysis of differentially expressed mRNAs. Table S4: screened coexpression pairs between DE lncRNAs and proliferation-related DE mRNAs based on the Pearson correlation coefficient. (Supplementary Materials)

\section{References}

[1] K. Zhang, Z.-M. Shi, Y.-N. Chang, Z.-M. Hu, H.-X. Qi, and W. Hong, "The ways of action of long non-coding RNAs in cytoplasm and nucleus," Gene, vol. 547, no. 1, pp. 1-9, 2014.

[2] C. P. Ponting, P. L. Oliver, and W. Reik, "Evolution and functions of long noncoding RNAs," Cell, vol. 136, no. 4, pp. 629-641, 2009.

[3] C. Lee and N. Kikyo, "Strategies to identify long noncoding RNAs involved in gene regulation," Cell \& Bioscience, vol. 2, no. 1, p. 37, 2012.

[4] A. N. Kallen, X.-B. Zhou, J. Xu et al., "The imprinted H19 lncRNA antagonizes let-7 microRNAs," Molecular Cell, vol. 52, no. 1, pp. 101-112, 2013.

[5] L. Yang, C. Lin, C. Jin et al., "IncRNA-dependent mechanisms of androgen-receptor-regulated gene activation programs," Nature, vol. 500, no. 7464, pp. 598-602, 2013.

[6] J. M. Engreitz, A. Pandya-Jones, P. McDonel et al., "The Xist lncRNA exploits three-dimensional genome architecture to spread across the X chromosome," Science, vol. 341, no. 6147, p. 1237973, 2013.

[7] W.-C. Liang, W.-M. Fu, C.-W. Wong et al., "The IncRNA H19 promotes epithelial to mesenchymal transition by functioning as miRNA sponges in colorectal cancer," Oncotarget, vol. 6, no. 26, pp. 22513-22525, 2015.

[8] Z. H. Bian, L. G. Jin, J. W. Zhang et al., "LncRNA-UCA1 enhances cell proliferation and 5-fluorouracil resistance in colorectal cancer by inhibiting miR-204-5p," Scientific Reports, vol. 6, no. 1, 2016.

[9] N. Fausto and J. S. Campbell, "The role of hepatocytes and oval cells in liver regeneration and repopulation," Mechanisms of Development, vol. 120, no. 1, pp. 117-130, 2003.

[10] N. Fausto, A. D. Laird, and E. M. Webber, "Liver regeneration. 2. Role of growth factors and cytokines in hepatic 
regeneration," The FASEB Journal, vol. 9, no. 15, pp. 15271536, 1995.

[11] C.-G. Huh, V. M. Factor, A. Sanchez, K. Uchida, E. A. Conner, and S. S. Thorgeirsson, "Hepatocyte growth factor/c-met signaling pathway is required for efficient liver regeneration and repair," Proceedings of the National Academy of Sciences, vol. 101, no. 13, pp. 4477-4482, 2004.

[12] D. Xu, F. Yang, J.-H. Yuan et al., "Long noncoding RNAs associated with liver regeneration 1 accelerates hepatocyte proliferation during liver regeneration by activating Wnt/ $\beta$-Catenin signaling," Hepatology, vol. 58, no. 2, pp. 739-751, 2013.

[13] Q. Gao, Y. Y. Gu, Y. N. Jiang et al., "Long non-coding RNA Gm2199 rescues liver injury and promotes hepatocyte proliferation through the upregulation of ERK1/2," Cell Death \& Disease, vol. 9, no. 6, 2018.

[14] L. L. Huang, S. S. Damle, S. Booten et al., "Partial hepatectomy induced long noncoding RNA inhibits hepatocyte proliferation during liver regeneration," PLoS One, vol. 10, no. 7, 2015.

[15] R. Dayoub, A. Vogel, J. Schuett et al., "Nrf2 activates augmenter of liver regeneration (ALR) via antioxidant response element and links oxidative stress to liver regeneration," Molecular Medicine, vol. 19, no. 1, pp. 237-244, 2013.

[16] M. A. Fernandez-Rojo, C. Restall, C. Ferguson et al., "Caveolin-1 orchestrates the balance between glucose and lipiddependent energy metabolism: implications for liver regeneration," Hepatology, vol. 55, no. 5, pp. 1574-1584, 2012.

[17] Y. Iimuro, T. Nishiura, C. Hellerbrand et al., "NFkappaB prevents apoptosis and liver dysfunction during liver regeneration," Journal of Clinical Investigation, vol. 101, no. 4, pp. 802-811, 1998.

[18] L. L. Hall, M. Byron, G. Pageau, and J. B. Lawrence, "AURKBmediated effects on chromatin regulate binding versus release of XIST RNA to the inactive chromosome," The Journal of Cell Biology, vol. 186, no. 4, pp. 491-507, 2009.

[19] R. Zhou, Y. Zhang, G. Du et al., "Down-regulated let-7b-5p represses glycolysis metabolism by targeting AURKB in asthenozoospermia," Gene, vol. 663, pp. 83-87, 2018.

[20] J.-P. Mallm and K. Rippe, "Aurora kinase B regulates telomerase activity via a centromeric RNA in stem cells," Cell Reports, vol. 11, no. 10, pp. 1667-1678, 2015.

[21] L. Xu, T. Liu, F. Han et al., "AURKB and MAPK involvement in the regulation of the early stages of mouse zygote development," Science China Life Sciences, vol. 55, no. 1, pp. 47-56, 2012.

[22] D. Cimini, X. Wan, C. B. Hirel, and E. D. Salmon, "Aurora kinase promotes turnover of kinetochore microtubules to reduce chromosome segregation errors," Current Biology, vol. 16, no. 17, pp. 1711-1718, 2006.

[23] J. Yang, T. Ikezoe, C. Nishioka, K. Udaka, and A. Yokoyama, "Bcr-Abl activates AURKA and AURKB in chronic myeloid leukemia cellsviaAKT signaling," International Journal of Cancer, vol. 134, no. 5, pp. 1183-1194, 2014.

[24] A. Madejón, J. Sheldon, I. Francisco-Recuero et al., "Hepatitis $\mathrm{C}$ virus-mediated Aurora B kinase inhibition modulates inflammatory pathway and viral infectivity," Journal of Hepatology, vol. 63, no. 2, pp. 312-319, 2015.

[25] A. Zekri, S. H. Ghaffari, M. Yaghmaie et al., "Inhibitor of aurora kinase B induces differentially cell death and polyploidy via DNA damage response pathways in neurological malignancy: shedding new light on the challenge of resistance to AZD1152-HQPA," Molecular Neurobiology, vol. 53, no. 3, pp. 1808-1823, 2016.
[26] K. B. Lee, H. Jin, S. Ye, B. H. Park, and S. M. Kim, "Recombinant human bone morphogenetic protein-2 inhibits gastric cancer cell proliferation by inactivating Wnt signaling pathway via c-Myc with aurora kinases," Oncotarget, vol. 7, no. 45 , pp. 73473-73485, 2016.

[27] L. B. Zhu, J. Jiang, X. P. Zhu et al., "Knockdown of Aurora-B inhibits osteosarcoma cell invasion and migration via modulating PI3K/Akt/NF-kappaB signaling pathway," International Journal of Clinical and Experimental Pathology, vol. 7, no. 7, pp. 3984-3991, 2014.

[28] D. Santamaría, C. Barrière, A. Cerqueira et al., " $\mathrm{Cdk} 1$ is sufficient to drive the mammalian cell cycle," Nature, vol. 448, no. 7155, pp. 811-815, 2007.

[29] O. Gavet and J. Pines, "Progressive activation of CyclinB1Cdk1 coordinates entry to mitosis," Developmental Cell, vol. 18, no. 4, pp. 533-543, 2010.

[30] G. Ira, A. Pellicioli, A. Balijja et al., "DNA end resection, homologous recombination and DNA damage checkpoint activation require CDK1," Nature, vol. 431, no. 7011, pp. 1011-1017, 2004.

[31] S. P. Wheatley, E. H. Hinchcliffe, M. Glotzer, A. A. Hyman, G. Sluder, and Y.-L. Wang, "CDK1 inactivation regulates anaphase spindle dynamics and cytokinesis in vivo," The Journal of Cell Biology, vol. 138, no. 2, pp. 385-393, 1997.

[32] T. Tsukahara, Y. Tanno, and Y. Watanabe, "Phosphorylation of the CPC by Cdk1 promotes chromosome bi-orientation," Nature, vol. 467, no. 7316, pp. 719-723, 2010.

[33] R. M. Golsteyn, "Cdk1 and Cdk2 complexes (cyclin dependent kinases) in apoptosis: a role beyond the cell cycle," Cancer Letters, vol. 217, no. 2, pp. 129-138, 2005.

[34] P. Liu, T. P. Kao, and H. Huang, "Cdk1 promotes cell proliferation and survival via phosphorylation and inhibition of FOXO1 transcription factor," Oncogene, vol. 27, no. 34, pp. 4733-4744, 2008.

[35] M. Pagano, "Control of DNA synthesis and mitosis by the Skp2-p27-Cdk1/2 axis," Molecular Cell, vol. 14, no. 4, pp. 414-416, 2004.

[36] W.-W. Huang, S.-C. Tsai, S.-F. Peng et al., "Kaempferol induces autophagy through AMPK and AKT signaling molecules and causes G2/M arrest via downregulation of CDK1/ cyclin B in SK-HEP-1 human hepatic cancer cells," International Journal of Oncology, vol. 42, no. 6, pp. 2069-2077, 2013.

[37] C. Preisinger, R. Körner, M. Wind, W. D. Lehmann, R. Kopajtich, and F. A. Barr, "Plk1 docking to GRASP65 phosphorylated by Cdk1 suggests a mechanism for Golgi checkpoint signalling," The EMBO Journal, vol. 24, no. 4, pp. 753-765, 2005.

[38] X. Q. Wang, C. M. Lo, L. Chen, E. S.-W. Ngan, A. Xu, and R. Y. Poon, "Cdk1-PDK1-PI3K/Akt signaling pathway regulates embryonic and induced pluripotency," Cell Death \& Differentiation, vol. 24, no. 1, pp. 38-48, 2017.

[39] L. L. Wu, B. Qu, Y. T. Qin et al., "Identification of cyclindependent kinase 1 as a novel regulator for controlling type I Interferon signaling in systemic lupus erythematosus," Arthritis \& Rheumatology, vol. 67, 2015.

[40] W. Gan, H. Zhao, T. G. Li, K. Liu, and J. Huang, "CDK1 interacts with iASPP to regulate colorectal cancer cell proliferation through p53 pathway," Oncotarget, vol. 8, no. 42, pp. 71618-71629, 2017.

[41] R. Visintin, S. Prinz, and A. Amon, "CDC20 and CDH1: a family of substrate-specific activators of APC-dependent proteolysis," Science, vol. 278, no. 5337, pp. 460-463, 1997. 
[42] E. R. Kramer, N. Scheuringer, A. V. Podtelejnikov, M. Mann, and J.-M. Peters, "Mitotic regulation of the APC activator proteins CDC20 and CDH1," Molecular Biology of the Cell, vol. 11, no. 5, pp. 1555-1569, 2000.

[43] H. Yu, "Cdc20: a WD40 activator for a cell cycle degradation machine," Molecular Cell, vol. 27, no. 1, pp. 3-16, 2007.

[44] Y. Yang, A. H. Kim, T. Yamada et al., "A Cdc20-APC ubiquitin signaling pathway regulates presynaptic differentiation," Science, vol. 326, no. 5952, pp. 575-578, 2009.

[45] M. V. Hadjihannas, D. B. Bernkopf, M. Brückner, and J. Behrens, "Cell cycle control of Wnt/ $\beta$-catenin signalling by conductin/axin2 through CDC20," EMBO Reports, vol. 13, no. 4, pp. 347-354, 2012.

[46] A.-H. Yen and J.-L. Yang, "Cdc20 proteolysis requires p38 MAPK signaling and Cdh1-independent APC/C ubiquitination during spindle assembly checkpoint activation by cadmium," Journal of Cellular Physiology, vol. 223, no. 2, pp. 327-334, 2010.

[47] Y. Ding, C. G. Hubert, J. Herman et al., "Cancer-Specific requirement for BUB1B/BUBR1 in human brain tumor isolates and genetically transformed cells," Cancer Discovery, vol. 3, no. 2, pp. 198-211, 2013.

[48] Y.-X. Shi, T. Zhu, T. Zou et al., "Prognostic and predictive values of CDK1 and MAD2L1 in lung adenocarcinoma," Oncotarget, vol. 7, no. 51, pp. 85235-85243, 2016.

[49] T. Song, Y. Zheng, Y. Wang et al., "Specific interaction of KIF11 with ZBP1 regulates the transport of -actin mRNA and cell motility," Journal of Cell Science, vol. 128, no. 5, pp. 1001-1010, 2015.

[50] F. Wang and S. L. Lin, "Knockdown of kinesin KIF11 abrogates directed migration in response to epidermal growth factor-mediated chemotaxis," Biochemical and Biophysical Research Communications, vol. 452, no. 3, pp. 642-648, 2014.

[51] Z. T. Swider, R. K. Ng, R. Varadarajan, C. J. Fagerstrom, and N. M. Rusan, "Fascetto (PRC1) interacting protein (FIP) ensures proper cytokinesis and ploidy," Molecular Biology of the Cell, vol. 30, no. 8, pp. 992-1007, 2019.

[52] C. Li, M. Lin, and J. Liu, "Identification of PRC1 as the p53 target gene uncovers a novel function of p53 in the regulation of cytokinesis," Oncogene, vol. 23, no. 58, pp. 9336-9347, 2004.

[53] H. W. Chaudhry, N. H. Dashoush, H. Tang et al., "Cyclin A2 mediates cardiomyocyte mitosis in the postmitotic myocardium," Journal of Biological Chemistry, vol. 279, no. 34, pp. 35858-35866, 2004.

[54] F. Yang, J. Gong, G. Wang, P. Chen, L. Yang, and Z. Wang, "Waltonitone inhibits proliferation of hepatoma cells and tumorigenesis via FXR-miR-22-CCNA2 signaling pathway," Oncotarget, vol. 7, no. 46, pp. 75165-75175, 2016.

[55] T. Gao, Y. Han, L. Yu, S. Ao, Z. Li, and J. Ji, "CCNA2 is a prognostic biomarker for ER+ breast cancer and tamoxifen resistance," PLoS One, vol. 9, no. 3, Article ID e91771, 2014.

[56] S. Dong, F. Huang, H. Zhang, and Q. Chen, "Overexpression of BUB1B, CCNA2, CDC20, and CDK1 in tumor tissues predicts poor survival in pancreatic ductal adenocarcinoma," Bioscience Reports, vol. 39, no. 2, Article ID BSR20182306, 2019.

[57] Y. Gan, Y. Li, T. Li, G. Shu, and G. Yin, "CCNA2 acts as a novel biomarker in regulating the growth and apoptosis of colorectal cancer," Cancer Management and Research, vol. 10, pp. 5113-5124, 2018.

[58] R. Broderick, J. Nieminuszczy, A. N. Blackford et al., "TOPBP1 recruits TOP2A to ultra-fine anaphase bridges to aid in their resolution," Nature Communications, vol. 6, no. 1, p. 6572, 2015.

[59] N. Wong, W. Yeo, W.-L. Wong et al., "TOP2A overexpression in hepatocellular carcinoma correlates with early age onset, shorter patients survival and chemoresistance," International Journal of Cancer, vol. 124, no. 3, pp. 644-652, 2009.

[60] J. Sun, Y. Du, Q. Song et al., "E2F is required for STAT3mediated upregulation of cyclin B1 and Cdc2 expressions and contributes to G2-M phase transition," Acta Biochimica et Biophysica Sinica, vol. 51, no. 3, pp. 313-322, 2019.

[61] J. Gu, X. Liu, J. Li et al., "MicroRNA-144 inhibits cell proliferation, migration and invasion in human hepatocellular carcinoma by targeting CCNB1," Cancer Cell International, vol. 19, no. 1, p. 15, 2019. 


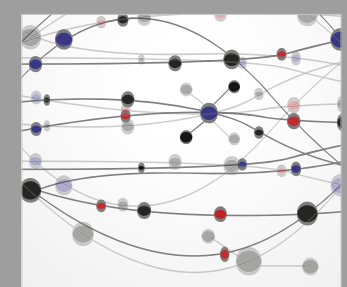

The Scientific World Journal
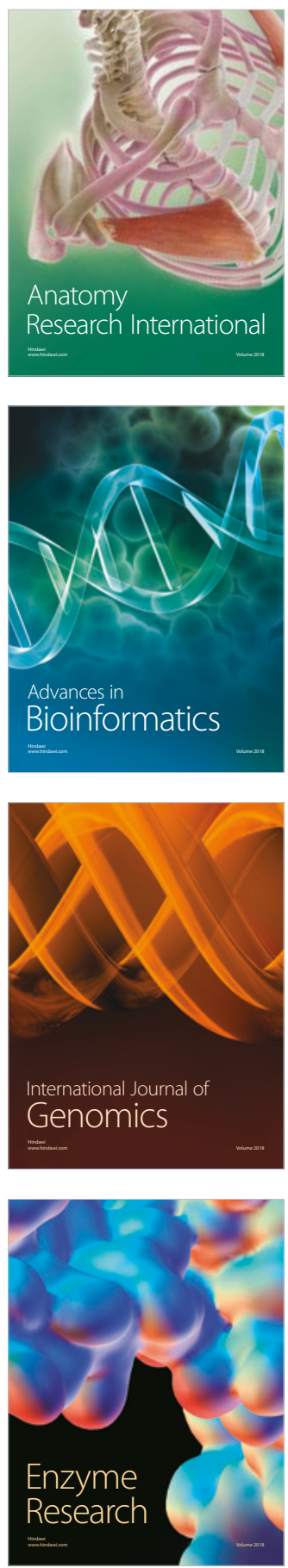
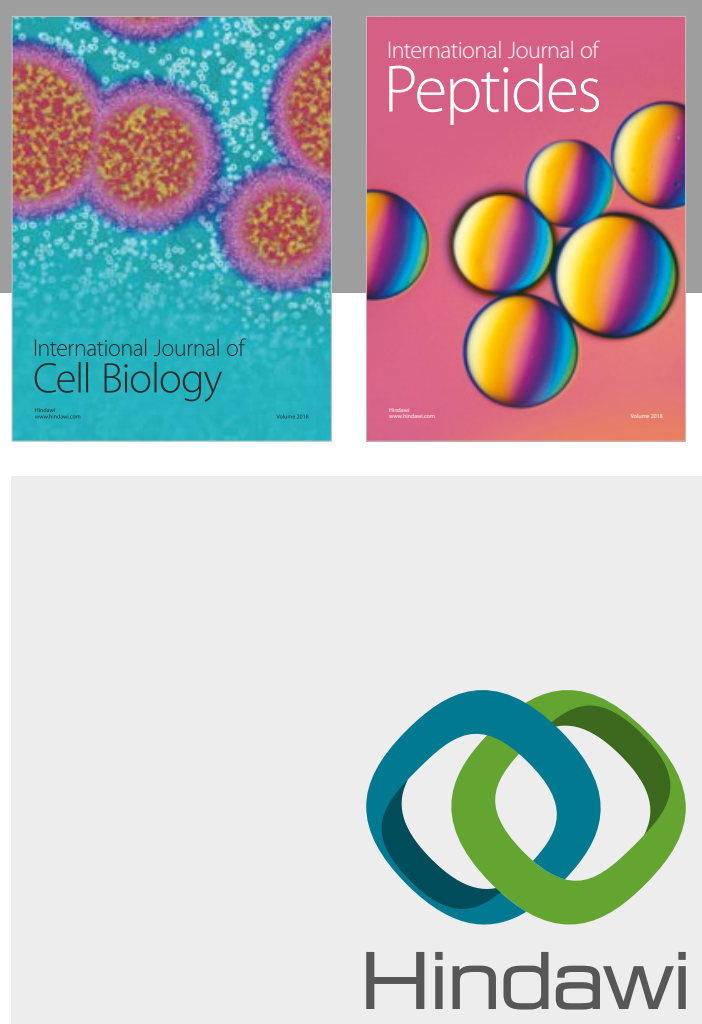

Submit your manuscripts at

www.hindawi.com
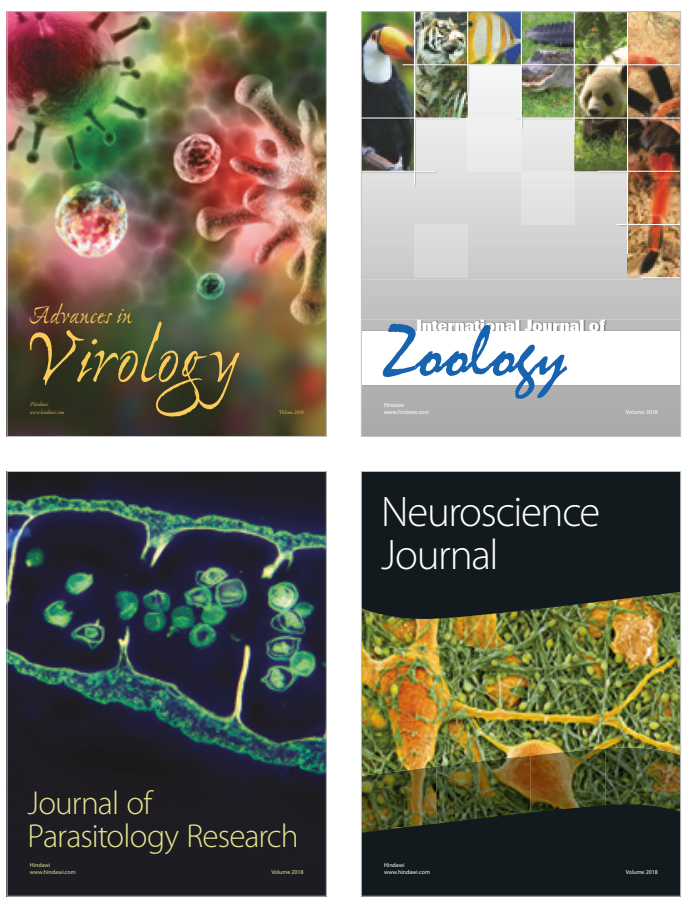
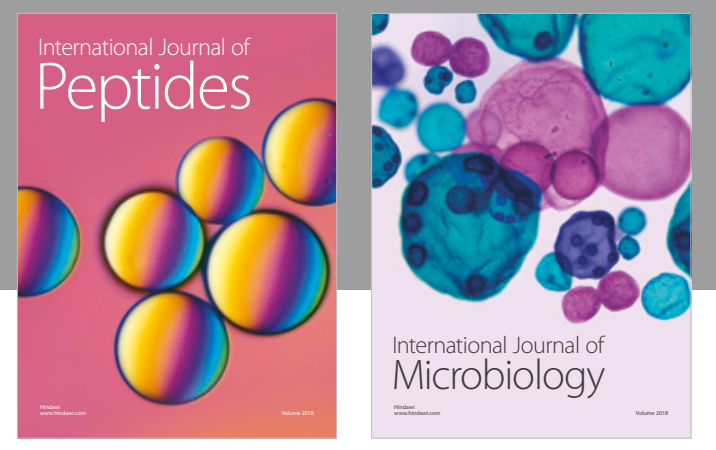

nternational Journal of Microbiology
Journal of
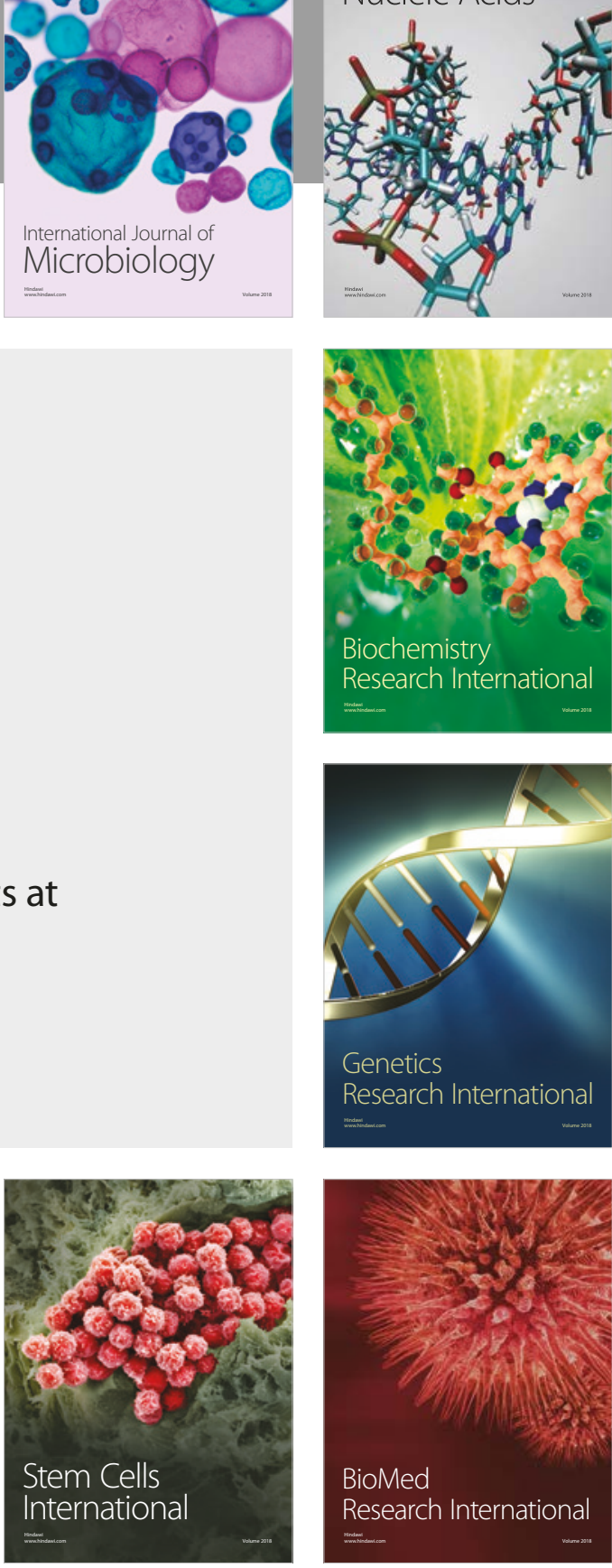
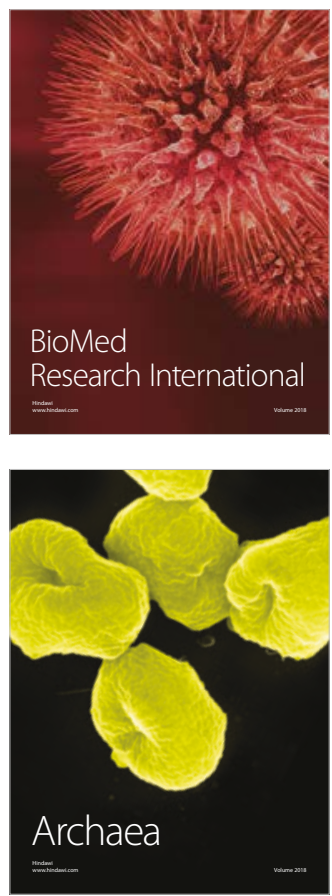